\title{
An Efficient Evolutionary Approach to Parameter Identification in a Building Thermal Model
}

\author{
Zhenyu Yang, Member, IEEE, Xiaoli Li, Chris P. Bowers, Thorsten Schnier, Member, IEEE, \\ Ke Tang, Member, IEEE, and Xin Yao, Fellow, IEEE
}

\begin{abstract}
Thermal models of buildings are often used to identify energy savings within a building. Given that a significant proportion of that energy is typically used to maintain building temperature, establishing the optimal control of the buildings thermal system is important. This requires an understanding of the thermal dynamics of the building, which is often obtained from physical thermal models. However, these models require detailed building parameters to be specified and these can often be difficult to determine. In this paper, we propose an evolutionary approach to parameter identification for thermal models that are formulated as an optimization task. A state-of-the-art evolutionary algorithm, i.e., SaNSDE +, has been developed. A fitness function is defined, which quantifies the difference between the energy-consumption
\end{abstract}

Manuscript received May 20, 2011; revised October 2, 2011; accepted October 16, 2011. Date of publication December 15, 2011; date of current version October 12, 2012. This work was supported in part by the National Natural Science Foundation of China under Grant U0835002, Grant 61028009, and Grant 61175065 , by the National Natural Science Foundation of Anhui Province under Grant 1108085J16, by the Engineering and Physical Sciences Research Council under Grant EP/F062567/1 on "Advanced Analysis of Building Energy Performance using Computational Intelligence Approaches," and by the European Union 7th Framework Program under Grant 247619. This paper was recommended by Associate Editor N. O. Attoh-Okine.

Z. Yang was with the Nature Inspired Computation and Applications Laboratory (NICAL), School of Computer Science and Technology, University of Science and Technology of China, Hefei 230026, China. He is now with the Department of Management Science and Engineering, College of Information Systems and Management, National University of Defense Technology, Changsha 410073, China (e-mail: zhyuyang@ mail.ustc.edu.cn).

$\mathrm{X}$. Li was with the Centre of Excellence for Research in Computational Intelligence and Applications (CERCIA), School of Computer Science, University of Birmingham, Edgbaston, Birmingham B15 2TT, U.K. He is now with the Department of Automation, Institute of Electrical Engineering, Yanshan University, Qinhuangdao 066004, China, and also with the National Key Laboratory of Cognitive Neuroscience and Learning, Beijing Normal University, Beijing 100875, China (e-mail: xiaoli.avh@gmail.com).

C. P. Bowers is with the Centre of Excellence for Research in Computational Intelligence and Applications (CERCIA), School of Computer Science, University of Birmingham, Birmingham B15 2TT, U.K. (e-mail: c.p.bowers@cs.bham.ac.uk).

T. Schnier is with the Centre of Excellence for Research in Computational Intelligence and Applications (CERCIA), School of Computer Science, University of Birmingham, Birmingham B15 2TT, U.K., and also with the Nature Inspired Computation and Applications Laboratory (NICAL), School of Computer Science and Technology, University of Science and Technology of China, Hefei 230026, China (e-mail: t.schnier@cercia.ac.uk).

$\mathrm{K}$. Tang is with the Nature Inspired Computation and Applications Laboratory (NICAL), School of Computer Science and Technology, University of Science and Technology of China, Hefei 230026, China (e-mail: ketang@ustc.edu.cn).

$\mathrm{X}$. Yao is with the Nature Inspired Computation and Applications Laboratory (NICAL), School of Computer Science and Technology, University of Science and Technology of China, Hefei 230026, China, and also with the Centre of Excellence for Research in Computational Intelligence and Applications (CERCIA), School of Computer Science, University of Birmingham, Edgbaston, Birmingham B15 2TT, U.K. (e-mail: x.yao@cs.bham.ac.uk).

Color versions of one or more of the figures in this paper are available online at http://ieeexplore.ieee.org.

Digital Object Identifier 10.1109/TSMCC.2011.2174983 time-series data that are derived from the identified parameters and that given by simulation with a set of predetermined target model parameters. In comparison with a conventional genetic algorithm, fast evolutionary programming, and two state-of-the-art evolutionary algorithms, our experimental results show that the proposed SaNSDE + has significantly improved both the solution quality and the convergence speed, suggesting this is an effective tool for parameter identification for simulated building thermal models.

Index Terms-Building thermal model, differential evolution (DE), evolutionary optimization, parameter identification.

\section{$\mathrm{DE}$}

SaNSDE SaNSDE+ JADE

SGA

FEP

PSO

CLPSO

NP

F

CR

$\mathrm{CRm}$

$\mathrm{CR}_{\text {rec }}$

$\Delta f_{\text {rec }}$

$p$

\section{NOMENCLATURE}

Differential evolution.

Self-adaptive DE with neighborhood search. Improved SaNSDE.

Adaptive DE variant.

Standard genetic algorithm.

Fast evolutionary programming.

Particle swarm optimization.

Comprehensive learning PSO.

Population size.

Scale factor of DE.

Crossover rate of DE.

Mean of Gaussian distribution for generating CR.

Recorded CR values for CRm updating.

Recorded fitness improvements.

Control parameter in the mutation of SaNSDE+.

\section{INTRODUCTION}

$\mathbf{E}$ NERGY consumption of buildings has steadily increased, reaching figures between $20 \%$ and $40 \%$ of the total energy consumed in developed countries [1]. Therefore, building energy efficiency is of prime concern and identifying energy savings beyond the obvious is becoming an increasingly important yet challenging task. Automated meter reading (AMR) systems, or smart meters, are often suggested as a solution. However, once the consumption data are captured, how to make use of these data to improve building energy efficiency is still an open issue.

One approach is to use the collected energy data to build a data-driven thermal model of the building, which can be used to estimate the thermal-energy demands of that building [2]. Comparison of the estimated demand with the metered consumption data can be a useful monitoring tool. Previous approaches to 
deriving thermal models of buildings have been proposed for electrical load estimation, such as [3]-[7]. These data-driven methods are not computationally efficient and do not easily lend themselves to building energy management and control systems since the results are often difficult to interpret.

In order to capture, within a simulation, i.e., the thermal dynamics apparent within a building, a reasonable account of the physical thermal behavior is required. Examples of simulation software that are intended for this purpose include ESP-r [8], TRNSYS [9], DOE-2 [10], BLAST, Energy Plus [11], TAS [12], FLUENT [13], and DEROB-LTH [14]. In this study, we use HAMBase [15], which is capable of simulating the indoor temperature, the indoor air humidity, and energy used for heating and cooling of a multizone building [16]. The program runs within the MATLAB environment.

A drawback of relying on these types of physical models is that they require a significant number of parameters, describing the characteristics of the building, to be defined in advance. Unfortunately, for most real buildings, many of these parameters are unknown and difficult to obtain, such as the characteristics of the construction materials.

In this study, we consider the identification of these thermal model parameters to be an optimization task, where the objective is to minimize the disparity between the estimated energy consumption of the model and the actual metered energy consumption of the building. This requires only energy consumption data to be collected from the buildings' AMR system.

Such a parameter-identification problem could be tackled with traditional optimization algorithms. However, most are gradient based and have the following drawbacks [17]:

1) they are often sensitive to the starting state;

2) they have a high probability of getting trapped at local optima for multimodal optimization problems;

3) they often require continuity and differentiability of the objective function.

The HAMBase model results in a nontrivial mapping from model parameters to the model energy consumption because of the dynamic nature of thermal models. This makes it very difficult to achieve the required continuity and differentiable characteristics. Therefore, a black-box optimization method is required, which avoids these drawbacks, such as an evolutionary optimization approach [18].

Evolutionary optimization methods utilize a populationbased stochastic search process and require little prior knowledge. Successful attempts have been suggested for many real-world applications [19], [20]. Specifically, in the study of heating, ventilating, and air conditioning (HVAC) system designs, genetic algorithms (GAs) have been applied to the optimization of HVAC system sizing [21], the simultaneous optimization of building construction, system supervisory control [22], and, most recently, the optimization of HVAC system configurations [23]. Related work has also been reported in the area of building model parameter identification [24]-[26]. However, rather than using an extensive physical model and considering it to be a black-box system, they mainly focused on the development of simplified building models. The

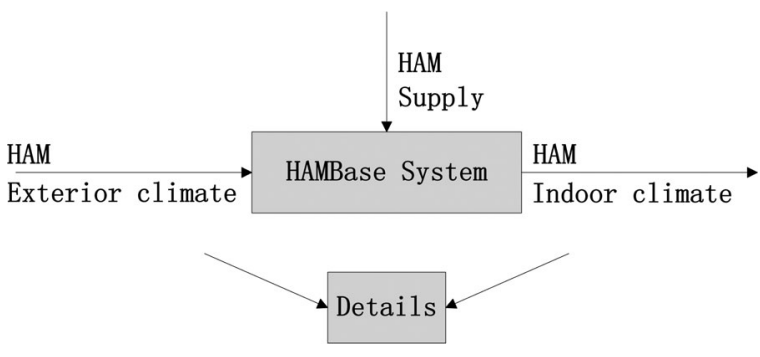

Fig. 1. Simple flowchart to demonstrate how the HAMBase model works.

evolutionary optimization methods that are used tend to be "off the shelf" generic algorithms.

In recent years, developments in evolutionary computation (EC) have led to many effective and efficient variants of evolutionary algorithms (EAs), which have been applied to numerous real-world applications. In the light of this, it seems that the application of evolutionary optimization deserves more investigation for the problem of parameter identification in thermal models of buildings.

In this paper, we discuss the application of a state-of-the-art EA, i.e., the SaNSDE [27], to parameter identification for a building thermal model. The efficacy of the SaNSDE algorithm has already been verified on a broad number of benchmark functions in [27] and [28]. Modifications to the original SaNSDE algorithm were made, by the introduction of a more efficient mutation strategy, to further improve its convergence speed for the parameter identification task that is described in this study. This improved SaNSDE variant is denoted as SaNSDE+.

For experimental validation, parameter identification problems were formulated using simulated building thermal models. The performance of the SaNSDE + algorithm, on this building thermal-model parameter identification task, is compared with ten variants of two well-known EAs, i.e., the SGA [29] and FEP [30], and two state-of-the-art EA variants, i.e., CLPSO [31] and JADE [32].

The rest of this paper is organized as follows. In Section II, we provide a brief overview about the adopted building thermal model. In Section III, we present the formulation and the fitness function for the parameter identification problem in building thermal models. In Section IV, we introduce the utilized optimization algorithms, i.e., SaNSDE and the new improved version $\mathrm{SaNSDE}+$. In Section V, we present the experimental studies. Finally, in Section VI, we conclude this paper with some remarks and topics for future work.

\section{OVERVIEW OF THE BUILDING THERMAL MODEL}

The utilized HAMBase ${ }^{1}$ is a physical model for the simulation of heat and vapor flows in a building. With the model, the indoor temperature, the indoor air humidity, and the energy use for heating and cooling of a multizone building can be simulated. A simple flowchart of the simulation process is demonstrated in Fig. 1.

\footnotetext{
${ }^{1}$ For a more detailed explanation of the HAMBase model see [33].
} 
In HAMBase, the smallest entity of the building is a zone, which can be one room but also a number of adjacent rooms with more or less equal climatic conditions. The model of a zone consists of a thermal model and a hygric model, and the main result of HAMBase is the thermal and hygric indoor climate and the energy use to maintain this climate. In order to develop such a model with desired simulation accuracy, the following issues were considered in HAMBase [15]:

1) heat and mass balance;

2) indoor climate model, which is further divided into the thermal indoor climate and the hygric indoor climate;

3) the envelope model, which is further divided into the thermal model and the hygric model;

4) fenestration, which includes the incident solar radiation, the heat flow through fenestration, the solar radiation absorbed in a zone, the daylight factor, and the airflow window;

5) air infiltration, which includes the simulation of airflows by natural ventilation that is caused by wind pressures and stack effects;

6) heating/cooling system, which includes the floor, walls, and ceiling heating or cooling systems;

7) mass sources, for which both the evaporation of liquid water and the inert gas are considered.

These issues, which are crucial in the construction of the whole HAMBase model, are basically implemented according to related physical principles. More detailed information about them can be found in [15].

To illustrate the complexity of the HAMBase model, we further describe how the heat and mass balance is handled. The thermal behavior of a zone in HAMBase depends on the following heat and moisture balance equations:

$$
\begin{aligned}
& \text { heat loss }+ \text { heat stored }=\text { heat gains }+ \text { auxiliary heat } \\
& \text { moisture loss }+ \text { moisture stored } \\
& \quad=\text { vapor production }+ \text { humidification }
\end{aligned}
$$

where:

1) heat loss is the transmitted and infiltrated energy losses through the envelope of a building;

2) heat stored is the energy stored by the building construction, which is related to the materials and size of the building;

3) heat gains are derived from the solar radiation, artificial lighting, domestic hot water, appliances, and so on;

4) auxiliary heat is supplied or extracted by a heating or cooling plant, respectively.

The moisture in the building is related to the diffusion and advection through the envelope, where:

1) moisture loss is the vapor losses by airflows;

2) moisture stored is the water held in porous materials;

3) vapor is produced by people, plants, appliances, etc;

4) humidification is needed for obtaining a relative humidity in the zone.

While these underlying equation are relatively simple, they interact with the state of the simulated building and external environment. This can generate significantly complex dynamic

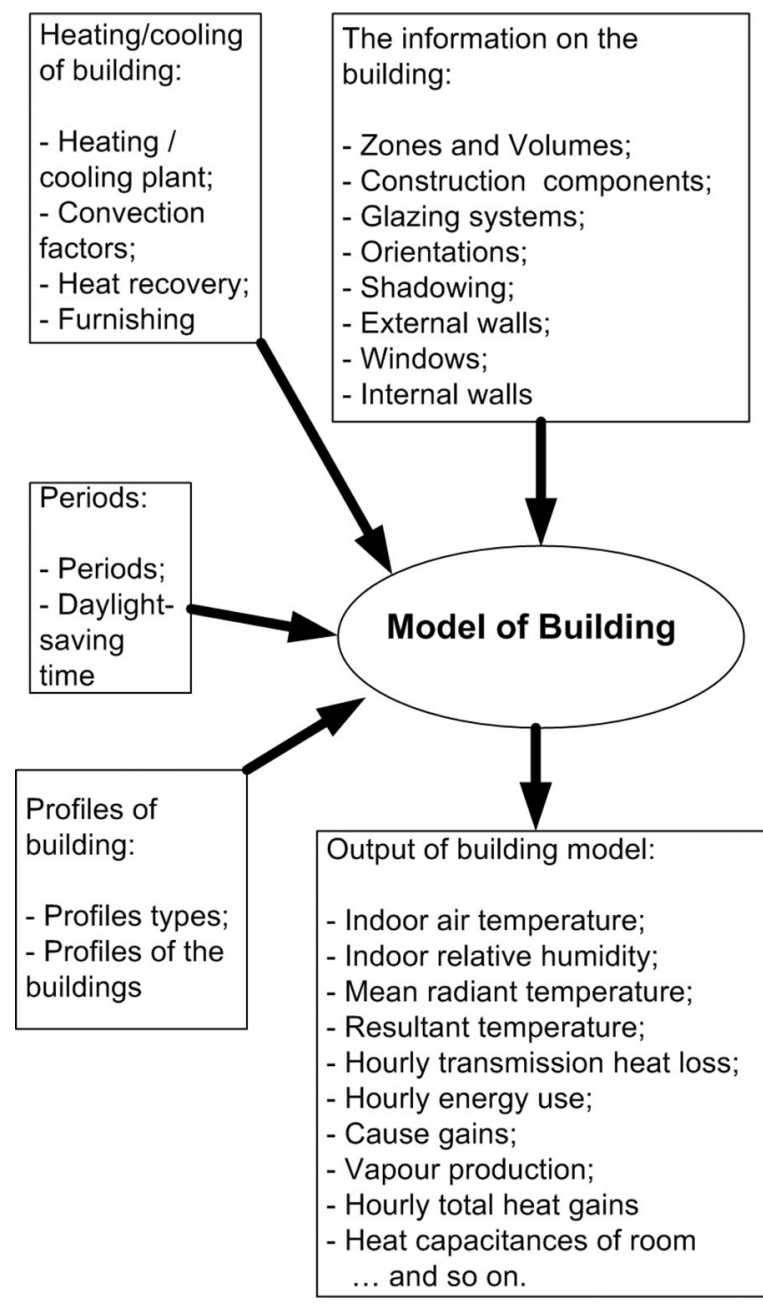

Fig. 2. Outline of the HAMBase for the building model.

behavior in terms of the times-series outputs that are produced from the simulation. An overview of the relevant inputs and outputs of the model is outlined in Fig. 2.

While some of the parameters are relatively easy to determine by direct measurement, such as heating/cooling plant behavior, zone topology, and volume, some are much more difficult to obtain, such as the building materials and occupant behavior. Given all required model parameters, the indoor temperature, the indoor air humidity, and energy consumption for heating and cooling of a multizone building can be obtained through simulation.

A reference model, which is included with the HAMBase software, is used, which provides sufficient complexity in operation while simplifying the process of development of a suitable model. Predetermined features of the reference model include the following:

1) simulation and operation periods for the building, occupants, and building systems;

2) construction and layout of the building into four zones;

3) performance and behavior of the HVAC components;

4) the properties of a finite set of materials that are used within the building include: material thickness [m], 
TABLE I

OBJective PaRAmeters of the BUILDING MOdel

\begin{tabular}{|c|c|c|}
\hline ID & Given & Description \\
\hline$x_{1}$ & 0.13 & $\begin{array}{l}\text { External wall: internal surface heat transfer resis- } \\
\text { tance }\left[\mathrm{Km}^{2} / \mathrm{W}\right]\end{array}$ \\
\hline$x_{2}$ & 0.04 & $\begin{array}{l}\text { External wall: surface heat transfer resistance at } \\
\text { the opposite site }\left[\mathrm{Km}^{2} / \mathrm{W}\right]\end{array}$ \\
\hline$x_{3}$ & 0.90 & $\begin{array}{l}\text { External wall: external solar radiation absorption } \\
\text { coefficient }\end{array}$ \\
\hline$x_{4}$ & 0.90 & External wall: external long-wave emissivity \\
\hline$x_{5}$ & 0.13 & $\begin{array}{l}\text { Internal wall: internal surface heat transfer resis- } \\
\text { tance }\left[\mathrm{Km}^{2} / \mathrm{W}\right]\end{array}$ \\
\hline$x_{6}$ & 0.13 & $\begin{array}{l}\text { Internal wall: surface heat transfer resistance at } \\
\text { the opposite site }\left[\mathrm{Km}^{2} / \mathrm{W}\right]\end{array}$ \\
\hline$x_{7}$ & 0.13 & $\begin{array}{l}\text { Roof construction: internal surface heat transfer } \\
\text { resistance }\left[\mathrm{Km}^{2} / \mathrm{W}\right]\end{array}$ \\
\hline$x_{8}$ & 0.04 & $\begin{array}{l}\text { Roof construction: surface heat transfer resistance } \\
\text { at the opposite site }\left[\mathrm{Km}^{2} / \mathrm{W}\right]\end{array}$ \\
\hline$x_{9}$ & 0.80 & $\begin{array}{l}\text { Roof construction: external solar radiation ab- } \\
\text { sorption coefficient }\end{array}$ \\
\hline$x_{10}$ & 0.90 & Roof construction: external long-wave emissivity \\
\hline
\end{tabular}

The actual values used in the simulation are listed in the Given column.

internal surface heat transfer resistance $\left[\mathrm{K} \cdot \mathrm{m}^{2} / \mathrm{W}\right]$, surface heat transfer resistance at the opposite site $\left[\mathrm{K} \cdot \mathrm{m}^{2} / \mathrm{W}\right]$, external solar radiation absorption coefficient, and external long-wave emissivity;

5) the meter sampling intervals for all measured time-series output variables of the simulation, which include temperature, relative humidity, and energy consumption. In this case, the sampling rate is set to $1 \mathrm{~h}$.

The key parameters of the HAMBase model we are concerned with, in this case, are listed in Table I. These represent the apparent material properties of the simulated building. These variables are chosen for parameter identification because of the difficulty in quantifying them in practice.

The reason we choose HAMBase as the simulation model is because it has several remarkable advantages. First, the designers based it on physics, which makes the model very realistic, instead of tuning it with results from a sophisticated transient model. Second, the simulation environment is transparent, and thus, the implementation of models is relatively easy [16]. Third, rather than commercial software, it facilitates open-source modeling, and all the simulation environment are publicly available from the website [33]. Although HAMBase might have its own limitations, such as numerous control parameters, too long simulation duration time, etc., it still is a practical platform to investigate the efficacy EAs for model parameter identification. Moreover, the optimization approach that is developed in this paper is taking the building model as a black box. It should not be model dependent and is expected to work for other models as well.

\section{PROBLEM FormULATION}

In order to formulate a parameter identification problem as an optimization task, an objective function must be defined. In this case, the objective is to minimize the disparity between the simulated energy consumption of the thermal model and the metered consumption of the real building by adjusting model parameters. In Fig. 3, an overview of the optimization process is shown.

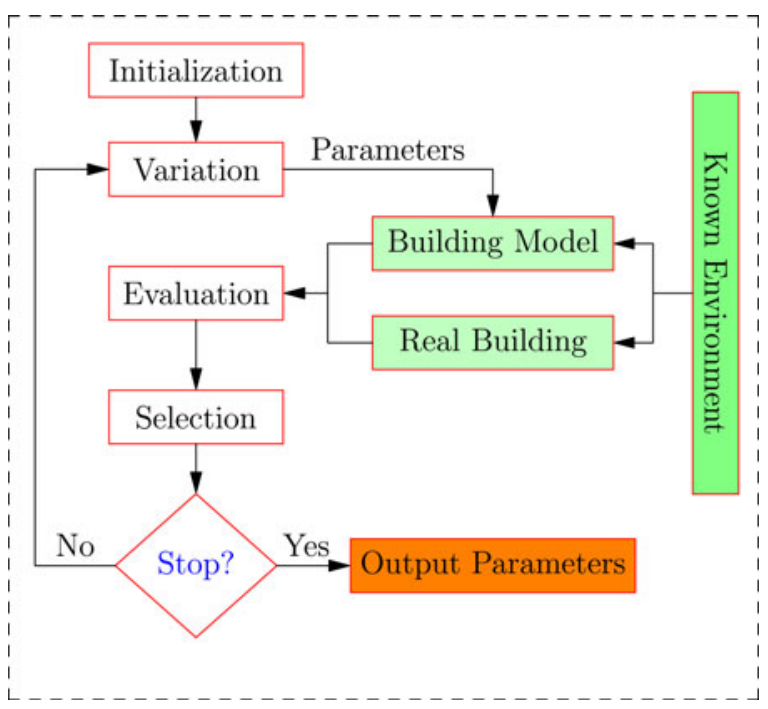

Fig. 3. Evolutionary optimization for the building thermal model parameter identification.

An initial population is formed from a set of randomly generated candidate parameters. Offspring are, then, generated by the application of perturbations to the candidate parameters of individuals in the current population. These offspring are evaluated based on a given fitness metric. After that, a selection strategy is used to identify a subset of the population to propagate to the next population for iteration until the stopping criterion is met.

This approach requires a clear and robust fitness evaluation (FE) method to be defined. For a given candidate solution, the basic idea of FE is to measure the deviation between the output of the real building, in the form of historical consumption and temperature data, and that of the simulated building model.

The behavior of a building could be considered to be a mapping from the time-dependent input $\mathbf{t}_{i}$ to the corresponding time-dependent output $\mathbf{y}\left(\mathbf{t}_{i}\right)$, where $i \in[1, T]$ is the index of time span. The input $\mathbf{t}_{i}$ could be the recorded climate data, such as the outdoor temperature, humidity, solar radiation, etc. The output $\mathbf{y}\left(\mathbf{t}_{i}\right)$ could be the energy consumption of the building, the internal temperature, humidity, etc., of each room in the building.

Similarly, a building model, which is designed to simulate the behavior of the real building, is also a mapping from the same input to output. The difference is that the building model is given in a parametrized form and its output depends on not only the input, but also a set of $n$ parameters, i.e., $\mathbf{x}=\left(x_{1}, x_{2}, \ldots, x_{n}\right) \in$ $\mathbb{R}^{n}$. Therefore, the building model output could be represented by $\hat{\mathbf{y}}\left(\mathbf{x}, \mathbf{t}_{\mathbf{i}}\right)$ at each time instant $\mathbf{t}_{i}, i \in[1, T]$. Given a set of identified parameters, the deviation between the output of the real building and that of the building model with the same input can be measured by the following function:

$$
f(\mathbf{x})=\frac{1}{T} \sum_{i=1}^{T}\left|\mathbf{y}\left(\mathbf{t}_{\mathbf{i}}\right)-\hat{\mathbf{y}}\left(\mathbf{x}, \mathbf{t}_{\mathbf{i}}\right)\right|^{p}
$$

where $p$ is a positive number. In practice, $p=1$ and $p=2$, which are called mean absolute error (MAE) and mean-squared error (MSE), respectively, are the most often used settings. Both 


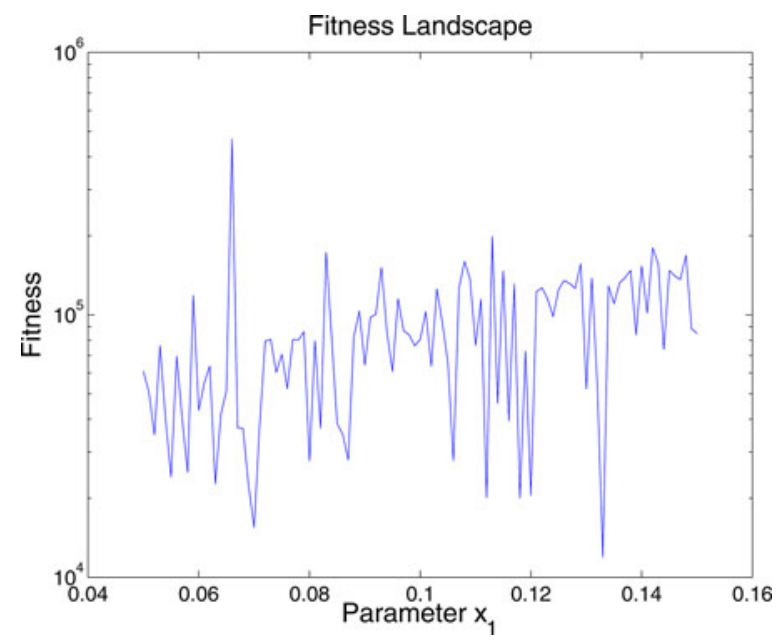

Fig. 4. Fitness landscape of the formulated problem against one of the model parameters.

MAE and MSE are suitable to serve as fitness functions, because they can reflect the goodness of a trial parameter solution. In this paper, we simply use the MAE, i.e., $p=1$, as the fitness function. It is possible that the fitness function $f(\cdot)$ is not noise free because of the observation noise in $\mathbf{y}\left(\mathbf{t}_{i}\right)$. Since much of the noise can be handled via general techniques, like standard filtering or statistical analysis [34], which is quite independent of the design of optimization algorithms, we will not consider noise in this study. Based on the reputation that EAs are effective in finding robust solutions in noisy environments [35], the proposed approach, in this paper, could be applicable to noise problems by the incorporation of a certain noise-handling technique.

Although the fitness function can be represented in such a simple form as shown in (1), it does not mean that the parameter identification problem is simple as well. To illustrate the characteristics of the fitness landscape, in Fig. 4, performance against one of the parameters (i.e., $x_{1}$ ) is shown, in the proximity of the optimal value (i.e., 0.13), with the other parameters randomly generated in the search space. Plots of performance against other parameters, and at different points in the search space, are similar: the fitness landscape is highly multimodal, and at the same time, there is highly interdependence between parameters.

One of the main factors that determines the difficulty of an optimization problem is the complexity of the mapping from the objective vector to the fitness value. In the parameter identification problem, the fitness mapping is determined by the objective parameter vector $\mathbf{x}$, and how the building thermal model transfers the model input $\mathbf{t}_{i}$ (climate data, such as outdoor temperature, humidity, solar radiation, etc.) to model output $\hat{\mathbf{y}}\left(\mathbf{x}, \mathbf{t}_{\mathbf{i}}\right)$ (energy consumption, indoor temperature, humidity, etc.). This means that the FE requires running simulation with other software (i.e., HAMBase). As discussed in Section I, the adopted HAMBase is a complex physical thermal model, and the mapping from model input to output is a thermodynamical simulation process. Since there is no explicit expression of the form of such a physical model simulation, it would be difficult, if not impossible, to utilize traditional optimization methods, for which the required gradient information is obviously not available.

With $f(\cdot)$ as the fitness function, the parameter identification problem is transferred into a bound-constrained multidimensional optimization problem as follows:

$$
\begin{aligned}
& \arg \min f(\mathbf{x}), \text { s.t. } \mathbf{x} \in S \\
& \mathbf{x}=\left(x_{1}, x_{2}, \ldots, x_{n}\right) \\
& S=\left\{\mathbf{x}: l_{i} \leq x_{i} \leq u_{i}, x_{i} \in \mathbb{R} \quad \forall i \in[1, n]\right\}
\end{aligned}
$$

where $l_{i}$ and $u_{i}$ denote the predefined upper bounds and the lower bounds of the $n$ parameters, respectively.

If we further consider the practical operational accuracy of these model parameters, a tolerance $e$ (e.g., $e=10^{-3}$ ) could be introduced to control the parameter solution accuracy. The tolerance is useful to increase search efficiency by the reduction of the overall size of the search space. With a predefined tolerance $e$, the original optimization problem can be transferred into an integer programming problem easily as follows:

$$
\begin{aligned}
& \arg \min f(\mathbf{x}), \text { s.t. } \mathbf{x}=e \cdot \mathbf{z}, \quad \mathbf{z} \in S^{\prime} \\
& \mathbf{z}=\left(z_{1}, z_{2}, \ldots, z_{n}\right) \\
& S^{\prime}=\left\{\mathbf{z}:\left\lfloor l_{i} / e\right\rfloor \leq z_{i} \leq\left\lceil u_{i} / e\right\rceil, z_{i} \in \mathbb{Z} \quad \forall i \in[1, n]\right\} .
\end{aligned}
$$

\section{IMPROVEd DifFERENTIAL EVolution Method}

\section{A. Classical Differential Evolution}

DE [36], [37] is a simple yet effective algorithm for numerical global optimization. It has shown excellent performance on a wide range of benchmark problems [38], [39] as well as many real-world applications [40], [41]. DE is distinguished by its novel mutation scheme. DE executes its mutation by adding a weighted difference vector between two individuals to a third individual. Then, the mutated individuals will be subject to discrete crossover and greedy selection with corresponding individuals of the last generation to produce offspring.

Individuals in $\mathrm{DE}$ are represented by $D$-dimensional vectors $\mathbf{x}_{i}, \forall i \in\{1, \cdots, \mathrm{NP}\}$, where $D$ is the number of objective parameters, and NP is the population size. According to [37], the classical DE can be summarized as follows.

1) Mutation:

$$
\mathbf{v}_{i}=\mathbf{x}_{r_{1}}+F \cdot\left(\mathbf{x}_{r_{2}}-\mathbf{x}_{r_{3}}\right)
$$

where $r_{1}, r_{2}, r_{3} \in[1, \mathrm{NP}]$ are random and mutually different integers, and they are also different with the vector index $i$. The scale factor $F>0$ is a real constant factor and is often set to 0.5 .

2) Crossover:

$$
\mathbf{u}_{i}(j)= \begin{cases}\mathbf{v}_{\mathbf{i}}(\mathbf{j}), & \text { if } U_{j}(0,1) \leq \mathrm{CR} \text { or } j=j_{\text {rand }} \\ \mathbf{x}_{i}(j), & \text { otherwise }\end{cases}
$$

where $U_{j}(0,1)$ stands for the uniform random number between 0 and 1 , and $j_{\text {rand }}$ is a randomly chosen index to ensure that the trial vector $\mathbf{u}_{i}$ does not duplicate $\mathbf{x}_{i}$. $\mathrm{CR} \in(0,1)$ is the crossover rate and often set to 0.9. 
3) Selection:

$$
\mathbf{x}_{i}^{\prime}= \begin{cases}\mathbf{u}_{\mathbf{i}}, & \text { if } f\left(\mathbf{u}_{i}\right) \leq f\left(\mathbf{x}_{i}\right) \\ \mathbf{x}_{i}, & \text { otherwise }\end{cases}
$$

where $\mathbf{x}_{i}^{\prime}$ is the offspring of $\mathbf{x}_{i}$ for the next generation. (Without loss of generality, we consider only the minimization problem in this paper).

There exist a number of DE variants based on different mutation strategies:

$$
\begin{aligned}
\mathbf{v}_{i} & =\mathbf{x}_{r_{1}}+F \cdot\left(\mathbf{x}_{r_{2}}-\mathbf{x}_{r_{3}}\right) \\
\mathbf{v}_{i} & =\mathbf{x}_{\text {best }}+F \cdot\left(\mathbf{x}_{r_{1}}-\mathbf{x}_{r_{2}}\right) \\
\mathbf{v}_{i} & =\mathbf{x}_{i}+F \cdot\left(\mathbf{x}_{\text {best }}-\mathbf{x}_{i}\right)+F \cdot\left(\mathbf{x}_{r_{1}}-\mathbf{x}_{r_{2}}\right) \\
\mathbf{v}_{i} & =\mathbf{x}_{\text {best }}+F \cdot\left(\mathbf{x}_{r_{1}}-\mathbf{x}_{r_{2}}\right)+F \cdot\left(\mathbf{x}_{r_{3}}-\mathbf{x}_{r_{4}}\right) \\
\mathbf{v}_{i} & =\mathbf{x}_{r_{1}}+F \cdot\left(\mathbf{x}_{r_{2}}-\mathbf{x}_{r_{3}}\right)+F \cdot\left(\mathbf{x}_{r_{4}}-\mathbf{x}_{r_{5}}\right)
\end{aligned}
$$

where $\mathbf{x}_{\text {best }}$ is the best individual in the current population.

Schemes (4) and (6), with notations as "DE/rand/1" and "DE/current-to-best/2", are the most often used in practice because of their good performance [37], [42].

Most recently, we proposed a new mutation strategy "DE/current-to-local-p-best/2" in [43] as follows:

$$
\mathbf{v}_{i}=\mathbf{x}_{i}+F \cdot\left(\mathbf{x}_{\text {lpbest }}-\mathbf{x}_{i}\right)+F \cdot\left(\mathbf{x}_{r_{1}}-\mathbf{x}_{r_{2}}\right)
$$

where $p \in(0,1]$, and $\mathbf{x}_{\text {lpbest }}$ is randomly chosen from a set $\mathcal{A}$ that is generated as follows at the beginning of each generation.

1) $\operatorname{Set} \mathcal{A}=\emptyset$.

2) Randomly assign each individual to $K$ groups, where $K=$ $\lfloor p * \mathrm{NP}\rfloor$, and NP is the population size.

3) Add the best individual in each group to $\mathcal{A}$.

The new "DE/current-to-local- $p$-best/2" is actually a generalization of the previous schemes, i.e., "DE/current-to-best/2" and "DE/current-to- $p$-best/2" [32]. Instead of using only the best individual, i.e., $\mathbf{x}_{\text {best }}$, the new mutation strategy adopts a set of superior individuals, i.e., $\mathbf{x}_{\text {lpbest }}$, to produce differential vectors. This will obviously increase the diversity of mutated individuals, and weaken the overwhelming influence of the current best individual $\mathbf{x}_{\text {best }}$. Based on the investigation in [43], DE with this mutation scheme is able to utilize the information of good solutions without the introduction of the high probability of being trapped by local optima, which is the main drawback of the other schemes that simply use $\mathbf{x}_{\text {best }}$.

\section{B. Improved Self-Adaptive Differential Evolution With Neighborhood Search}

As presented in Section IV-A, the conventional DE has several candidate mutation schemes, and three control parameters, i.e., population size NP, scale factor $F$, and crossover rate CR. Its performance is often highly dependent on the used mutation scheme and parameter settings. Apart from the parameter NP (which is common for all population-based algorithms), mutation strategy selection, parameters $F$, and CR adaptations are the three most important issues of DE research. Much work has been done along these lines. The relationship between the control parameters and population diversity has been analyzed in [44].
Experimental parameter studies and empirical parameter settings of DE have been carried out in [45] and [46]. Self-adaptive strategies have also been investigated to adapt these control parameters [47], as well as different mutation schemes [42].

In [27], we proposed a new DE variant, namely SaNSDE, to adapt the two control parameters, i.e., $F$ and $\mathrm{CR}$, and the choice of mutation schemes. Three self-adaptive mechanisms are utilized in SaNSDE, which are summarized as follows.

1) Self-adaptive selection of mutation scheme based on two candidates, where SaNSDE utilizes mutation schemes (4) and (6) as candidates and produces the trial vector based on

$$
\mathbf{v}_{i}= \begin{cases}\text { Equation }(4), & \text { if } U_{i}(0,1)<p \\ \text { Equation }(6), & \text { otherwise. }\end{cases}
$$

Initially, the parameter $p$ is set to 0.5 . After evaluation of all offspring, the number of offspring successfully entering the next generation, while generated by (4) and (6), are recorded as $n s_{1}$ and $n s_{2}$, respectively, and the numbers of offspring discarded, while generated by (4) and (6), are recorded as $n f_{1}$ and $n f_{2}$. Those two pairs of numbers are accumulated within a specified number of generations (50 in SaNSDE), called the "learning period." Then, the probability $p$ is updated according to

$$
p=\frac{n s_{1} \cdot\left(n s_{2}+n f_{2}\right)}{n s_{2} \cdot\left(n s_{1}+n f_{1}\right)+n s_{1} \cdot\left(n s_{2}+n f_{2}\right)} .
$$

Here, $n s_{1}, n s_{2}, n f_{1}$, and $n f_{2}$ will be reset once $p$ is updated after each learning period.

2) Self-adaptation of scale factor $F$ using either the Gaussian or the Cauchy distribution. SaNSDE generates $F_{i}$ for each individual based on

$$
F_{i}= \begin{cases}N_{i}(0.5,0.3), & \text { if } U_{i}(0,1)<f p \\ C_{i}(0,1), & \text { otherwise }\end{cases}
$$

where $f p$ is self-adapted as $p$ is done according to (11), except that here we have to record the corresponding $F$ values, which make offspring enter the next generation successfully. $N_{i}(0.5,0.3)$ denotes a Gaussian random number with mean 0.5 and standard deviation $0.3 . C_{i}(0,1)$ denotes a Cauchy random number with the location parameter 0 and scale parameter 1 .

3) Self-adaptive crossover rate $\mathrm{CR}$ with the Gaussian distribution. SaNSDE allocates $\mathrm{CR}_{i}$ for each individual according to

$$
\mathrm{CR}_{i}=N_{i}(\mathrm{CRm}, 0.1) .
$$

Here, CRm is, initially, set to 0.5 . These $\mathrm{CR}$ values for all individuals remain the same for several generations ( 5 in SaNSDE), and then, a new set of CR values is generated using the same equation. During every generation, the $\mathrm{CR}$ values that are associated with offspring successfully entering the next generation are recorded in an array $\mathrm{CR}_{\mathrm{rec}}$, and the corresponding improvement on fitness values are also recorded in another array $\Delta f_{\text {rec }}$. After a specified number of generations (25 in SaNSDE), CRm will be 
updated:

$$
\begin{aligned}
\mathrm{CRm} & =\sum_{k=1}^{\left|\mathrm{CR}_{\mathrm{rec}}\right|} w_{k} * \mathrm{CR}_{\mathrm{rec}}(k) \\
w_{k} & =\Delta f_{\mathrm{rec}}(k) /\left(\sum_{k=1}^{\left|\Delta f_{\mathrm{rec}}\right|} \Delta f_{\mathrm{rec}}(k)\right)
\end{aligned}
$$

where $\mathrm{CR}_{\text {rec }}$ and $\Delta f_{\text {rec }}$ will be reset once $\mathrm{CRm}$ is updated.

Since the FE of a simulated building energy model can be computationally expensive, the convergence speed of EAs for parameter identification is very important. To further increase the convergence speed of SaNSDE, we revise its self-adaptation mechanisms as follows.

1) The candidate mutation scheme (6) is replaced with the most recent one (9), i.e.,

$$
\mathbf{v}_{i}= \begin{cases}\operatorname{Equation}(4), & \text { if } U_{i}(0,1)<p \\ \text { Equation }(9), & \text { otherwise. }\end{cases}
$$

2) The Cauchy random number $C_{i}(0,1)$ in (12) is replaced with $C_{i}(0.5,0.3)$, because it was found that $F$ values around 0.5 are more likely to produce successful mutation [45].

This new improved variant is denoted as SaNSDE+. Both SaNSDE and SaNSDE + will be applied to the parameter identification problems that are formulated in Section III. To make them able to operate in integer search space, slight modification is performed by rounding off the real parameter values to the nearest integers [48]. The integer version of these two algorithms are denoted as [SaNSDE] and [SaNSDE+].

\section{EXPERIMENTAL STUDIES}

\section{A. Setup}

In this study, we investigate the application of evolutionary methods (e.g., SaNSDE and SaNSDE+) for parameter identification of a building thermal model. In order to determine the performance of this approach for a real building, we would need to know the correct value of the relevant building parameters, to ensure that the parameters that are identified using the thermal model correspond correctly. For a real world building, this would require the collection of both historical consumption data and the corresponding values for the model parameters. This is a costly and time-consuming process, which is beyond the scope of this study.

Therefore, a simulation instance of the adopted building model is used as the target. The instance building can be achieved easily by providing a set of valid parameter values for the building model.

The target instance building has four zones, which is the typical setting of a basic building simulation. The inputs of the building simulation are the outside temperatures and humidities for a period of time (e.g., $1 \mathrm{mo}$ ). The outputs of the building simulation includes the temperatures and humidities of all the zones, and the total energy consumption of the building corresponding to the input time period. The I/O data were taken from
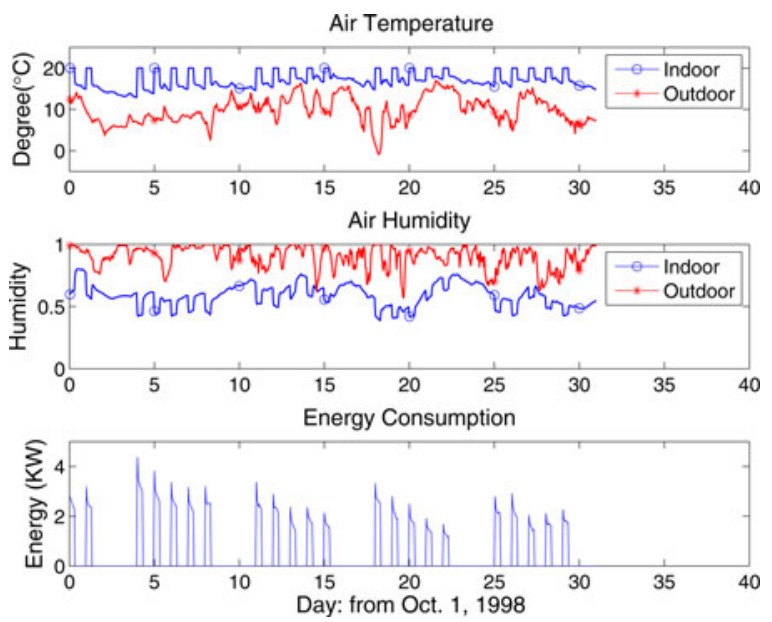

Fig. 5. I/O data of the target instance building, including the outdoor and indoor (only the first room is shown) temperature and humidity, and energy consumption of the building.

a time period of $1 \mathrm{mo}^{2}$ real climate data of De Bilt starting from the Oct. 1, 1998, which can be seen from Fig. 5. The data were collected at discrete hourly time steps. This resulted in a total of $31 * 24=744$ data points.

The objective parameters of the building model and their physical significance can be found in Table I. Based on the given objective parameters, two problems with different complexities can be formulated as follows.

1) The six-parameter problem: optimizing only the first six parameters in Table I, with the remaining parameters being predefined as their optimal values.

2) The ten-parameter problem: optimizing all the ten listed parameters.

As for the parameter settings of the four adopted DE variants (i.e., SaNSDE, SaNSDE+, [SaNSDE], and [SaNSDE+]), population size $\mathrm{NP}=50$ was used for all of them. The probability for choosing local "pbest" was set to $p=0.1$ in [SaNSDE+] and SaNSDE + [43]. The number of generations was set to 1000 for the six-parameter problem, and 2000 for the ten-parameter problem. The search ranges for all objective parameters were set to $\left\{x_{i} \in[0,1] \forall i \in[1,10]\right\}$.

The initial population was generated randomly in the search space. The tolerance for integer programming was set to $e=10^{-3}$, which means the corresponding search space was transferred to

$$
\left\{x_{i}=z_{i} * e, z_{i} \in[0,1000] \forall i \in[1,10]\right\}
$$

for $[\mathrm{SaNSDE}]$ and $[\mathrm{SaNSDE}+]$.

\section{B. Results}

The simulation results over 30 independent runs of the four tested DE variants on the six-parameter and ten-parameter problems are summarized in Tables III and IV, respectively. In these tables, smaller values indicate better performance than larger

\footnotetext{
${ }^{2}$ Validation with large dataset $(9 \mathrm{mo})$ was provided in Section V-D.
} 
TABLE II

Best Parameter SOlution Found By EACH Algorithm

\begin{tabular}{|c|c|c|c|c|}
\hline ID/Given & [SaNSDE] & [SaNSDE+] & SaNSDE & SaNSDE+ \\
\hline$x_{1} / 0.13$ & 0.13 & 0.13 & 0.13 & 0.13 \\
\hline$x_{2} / 0.04$ & 0.04 & 0.04 & 0.04 & 0.04 \\
\hline$x_{3} / 0.90$ & 0.90 & 0.90 & 0.90 & 0.90 \\
\hline$x_{4} / 0.90$ & 0.90 & 0.90 & 0.90 & 0.90 \\
\hline$x_{5} / 0.13$ & 0.13 & 0.13 & 0.13 & 0.13 \\
\hline$x_{6} / 0.13$ & 0.13 & 0.13 & 0.13 & 0.13 \\
\hline$x_{7} / 0.13$ & 0.13 & 0.13 & 0.13 & 0.13 \\
\hline$x_{8} / 0.04$ & 0.04 & 0.04 & 0.0399999 & 0.0399999 \\
\hline$x_{9} / 0.80$ & 0.80 & 0.80 & 0.800001 & 0.800001 \\
\hline$x_{10} / 0.90$ & 0.90 & 0.90 & 0.900001 & 0.900001 \\
\hline
\end{tabular}

TABLE III

Simulated Results on the SiX-Parameter Problem Over 30 Runs

\begin{tabular}{|l|c|c|c|c|}
\hline & {$[$ SaNSDE $]$} & {$[$ SaNSDE+ $]$} & SaNSDE & SaNSDE+ \\
\hline Best & $\mathbf{0 . 0 0 e + 0 0}$ & $\mathbf{0 . 0 0 e + 0 0}$ & $2.07 \mathrm{e}-08$ & $1.45 \mathrm{e}-08$ \\
\hline Median & $1.62 \mathrm{e}-03$ & $\mathbf{0 . 0 0 e + 0 0}$ & $3.39 \mathrm{e}-08$ & $1.96 \mathrm{e}-08$ \\
\hline Worst & $4.82 \mathrm{e}-02$ & $1.41 \mathrm{e}-02$ & $6.54 \mathrm{e}-08$ & $\mathbf{2 . 4 2 e - 0 8}$ \\
\hline Mean & $6.81 \mathrm{e}-03$ & $5.59 \mathrm{e}-04$ & $3.55 \mathrm{e}-08$ & $\mathbf{1 . 9 9 e - 0 8}$ \\
\hline Std & $1.09 \mathrm{e}-02$ & $2.60 \mathrm{e}-03$ & $8.71 \mathrm{e}-09$ & $\mathbf{2 . 6 4 e - 0 9}$ \\
\hline SR & $14 / 30$ & $28 / 30$ & $\mathbf{3 0 / 3 0}$ & $\mathbf{3 0 / 3 0}$ \\
\hline
\end{tabular}

"SR" means the successful runs over the 30 runs.

TABLE IV

Simulated Results on the TEN-PARAMETER PRoblem Over 30 RunS

\begin{tabular}{|l|c|c|c|c|}
\hline & {$[$ SaNSDE $]$} & {$[$ SaNSDE +$]$} & SaNSDE & SaNSDE+ \\
\hline Best & $\mathbf{0 . 0 0 e + 0 0}$ & $\mathbf{0 . 0 0 e + 0 0}$ & $7.16 \mathrm{e}-08$ & $5.40 \mathrm{e}-08$ \\
\hline Median & $3.17 \mathrm{e}-02$ & $4.52 \mathrm{e}-03$ & $8.95 \mathrm{e}-08$ & $7.86 \mathrm{e}-08$ \\
\hline Worst & $8.09 \mathrm{e}-02$ & $6.36 \mathrm{e}-02$ & $4.98 \mathrm{e}-02$ & $\mathbf{1 . 7 1 e - 0 7}$ \\
\hline Mean & $3.51 \mathrm{e}-02$ & $1.60 \mathrm{e}-03$ & $2.82 \mathrm{e}-03$ & $\mathbf{8 . 9 6 e - 0 8}$ \\
\hline Std & $2.51 \mathrm{e}-02$ & $2.02 \mathrm{e}-02$ & $1.01 \mathrm{e}-02$ & $\mathbf{3 . 1 7 e - 0 8}$ \\
\hline SR & $5 / 30$ & $12 / 30$ & $27 / 30$ & $30 / 30$ \\
\hline
\end{tabular}

"SR" means the successful runs over 30 runs.

values, because the fitness function was given based on the error of the building thermal model. Statistical indicators, such as "Best," "Median," "Mean," "Worst," and "Std" (standard deviation) values over 30 independent runs, are provided to ensure a fair comparison of performance between the tested algorithms.

A run is regarded as successful if the final fitness value is 0 for integer programming, or smaller than $10^{-5}$ for real-coded EA. The ratio of successful runs is denoted by "SR."

For each algorithm the best fitness achieved is very small or 0 but we can validate these model parameters themselves by comparing them to the instance parameters used in the original simulation.

This is shown in Table II, in which the best parameter solutions found by each of the algorithms on the ten-parameter problem are listed. The real-coded SaNSDE and SaNSDE+ found slightly different parameter values for $x_{8}, x_{9}$, and $x_{10}$. [SaNSDE] and [SaNSDE +$]$ provided the more accurate results because the search accuracy of them were limited at the level of $e=10^{-3}$.

For the results of six-parameter problem in Table III, [SaNSDE] were trapped in local optimum frequently $(\mathrm{SR}=$ 14/30), while [SaNSDE+] found the optimal solution with much better probability ( $\mathrm{SR}=28 / 30$ ). There is not much difference between SaNSDE and SaNSDE+ on this problem. However, for the more complex ten-parameter problem in Table IV, the difference between them are more significant. [SaNSDE] ( $\mathrm{SR}=5 / 30)$ is more likely to be trapped in local optima than $[\mathrm{SaNSDE}+](\mathrm{SR}=12 / 30)$. For the real-coded algorithms, SaNSDE + can still find the near-optimum consistently, but the successful rate of SaNSDE dropped to $\mathrm{SR}=27 / 30$.

The evolution curves of these algorithms are also provided in Fig. 6. The convergence speed of SaNSDE + and [SaNSDE+] appears to be generally faster than SaNSDE and [SaNSDE], respectively. This confirms that the proposed improvements in SaNSDE+ work well. Another interesting point is that realcoded algorithms often converged faster than the integer programming algorithms. This might be because $\mathrm{DE}$ is originally designed for real-coded optimization, and the method of discretizing the parameter values into an integer domain influences their efficiency.

Based on the achieved results, SaNSDE+ is the most recommended one among all the tested evolutionary optimization methods. It can be concluded that the proposed SaNSDE+ is sufficiently capable of the parameter identification problems in building thermal models.

\section{Comparison With Classical EAs}

To further verify the efficacy of our method, we also compared the performance of SaNSDE + with two other well-known EAs, i.e., SGA [29] and FEP [30]. Many former studies have demonstrated that they are the appropriate algorithms for evolutionary parameter identification. In the literature [25], [26], GA was used to estimate the parameters of some simplified building models using the operation data collected from site monitoring. Various case studies were conducted to validate the efficacy of the parameter optimization method. As for FEP, in [49], it was successfully used to determine unified creep damage constitutive equations, which were formulated as a complex, nonconvex, and nondifferentiable global optimization problem. In [50], in order to obtain a reasonably good description of the luminosity profiles of galaxies, FEP was used to fit the parameters for functional forms identified in the previous step. The proposed approach has been applied to modeling 18 elliptical galaxies profiles.

Since both the two algorithms have some control parameters, which may affect their performance greatly, we tested several variants of them with different parameter settings. For the SGA, the control parameters are the chromosome length $L$ for each variable, crossover rate $\mathrm{CR}$, mutation probability "pm," and two candidate selection schemes, i.e., stochastic universal sampling (SUS) and roulette wheel selection (RWS). According to [51], we set $\mathrm{CR}=0.7$, and $\mathrm{pm}=0.7 / L$. Other parameters were tuned to formulate the following four different SGA variants.

1) SGA-10S: Using length $L=10$, and the SUS selection scheme. $L=10$ implies the accurate level of objective parameters is $10^{-3}$, which is consistent with our setting for the integer programming problem.

2) SGA-10R: Using length $L=10$, and the RWS selection scheme.

3) SGA-20S: Using length $L=20$, and the SUS selection scheme. Setting $L$ to 20 is likely to generate more accurate 

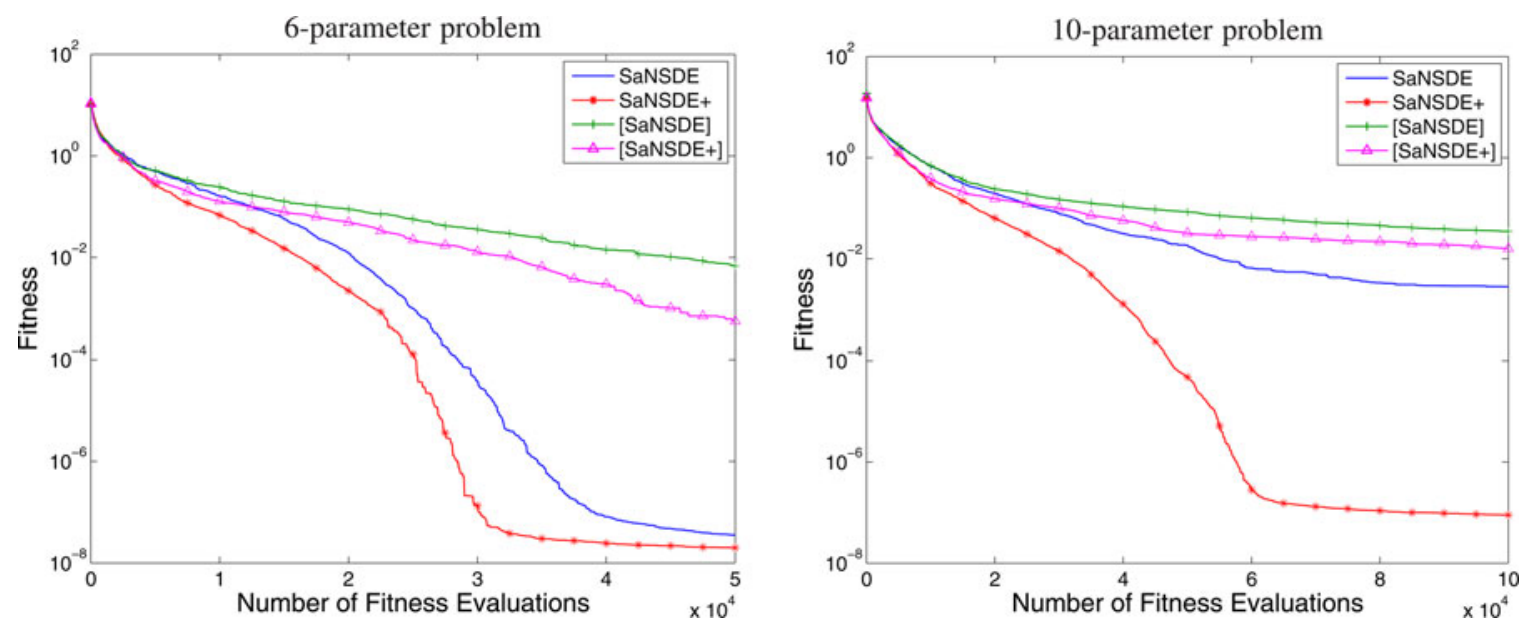

Fig. 6. Evolution curves for the six-parameter (left) and ten-parameter (right) problems. All results have been averaged over 30 independent runs.

results and makes the algorithm act like real-coded ones (e.g., SaNSDE+).

4) $S G A-20 R$ : Using length $L=20$, and the RWS selection scheme.

For FEP, the control parameters are the tournament selection size $q$, and the initial strategy parameter $\eta$. It was also claimed that a small lower bound for controlling strategy parameters [52] and swapping the order of variating objective variables and strategy parameters may improve FEP's performance [30]. As suggested in the original FEP algorithm, we set $\eta=3.0$ and tuned other parameters, which resulted in the following six FEP variants.

1) FEP-2N, FEP-5N, and FEP-10N: The original FEP with $q=2,5,10$ as the tournament size, respectively. No other strategy was used to further improve FEP.

2) FEP-2Y, FEP-5Y, and FEP-10Y: FEP with $q=2,5,10$ as the tournament size, respectively. Swapping the order of variating objective variables and strategy parameters, and using $10^{-4}$ as the lower bound for strategy parameters.

For SaNSDE+, since most of its parameters are self-adapted, we only need to set $p=0.1$, which is the probability for choosing local "pbest."

For all the tested algorithms, NP $=50$ was used as the population size. To provide a fair comparison, the same number of FEs was used for them. The code of SGA was taken from the publicly available toolbox GATBX [51], while the other algorithm FEP was implemented based on the description in [30].

The results of all the algorithms on the ten-parameter problem are summarized in Table V. It is obvious that the results of SaNSDE + are significantly better than that of all SGA and FEP variants. The best variants of FEP and SGA on average are FEP-5Y and SGA-10S, respectively. However, with $10^{-5}$ as the success fitness threshold, the best results of them are still far away from the optimum, while SaNSDE+ always found the optimum in the tested 30 independent runs. The parameter solutions that are presented in Table VI also confirm that FEP and SGA are not able to identify some of the objective parameters accurately. From the evolution curves that are presented in Fig. 7, it can be found that SGA and FEP stagnated rather
TABLE V

COMPARISON AMONG ALGORITHMS ON THE TEN-PARAMETER PROBLEM OVER 30 RUNS

\begin{tabular}{|l|c|c|c|c|c|}
\hline & Best & Mean & Worst & Std & SR \\
\hline FEP-2N & $3.38 \mathrm{e}-02$ & $7.59 \mathrm{e}-01$ & $3.38 \mathrm{e}+00$ & $9.30 \mathrm{e}-01$ & $0 / 30$ \\
\hline FEP-5N & $3.70 \mathrm{e}-02$ & $6.27 \mathrm{e}-01$ & $2.88 \mathrm{e}+00$ & $7.41 \mathrm{e}-01$ & $0 / 30$ \\
\hline FEP-10N & $3.41 \mathrm{e}-02$ & $6.03 \mathrm{e}-01$ & $3.04 \mathrm{e}+00$ & $8.14 \mathrm{e}-01$ & $0 / 30$ \\
\hline FEP-2Y & $2.49 \mathrm{e}-02$ & $3.86 \mathrm{e}-01$ & $2.03 \mathrm{e}+00$ & $6.60 \mathrm{e}-01$ & $0 / 30$ \\
\hline FEP-5Y & $2.85 \mathrm{e}-02$ & $2.33 \mathrm{e}-01$ & $2.02 \mathrm{e}+00$ & $3.95 \mathrm{e}-01$ & $0 / 30$ \\
\hline FEP-10Y & $2.17 \mathrm{e}-02$ & $4.67 \mathrm{e}-01$ & $2.01 \mathrm{e}+00$ & $6.77 \mathrm{e}-01$ & $0 / 30$ \\
\hline SGA-10S & $1.49 \mathrm{e}-01$ & $8.58 \mathrm{e}-01$ & $2.97 \mathrm{e}+00$ & $7.69 \mathrm{e}-01$ & $0 / 30$ \\
\hline SGA-10R & $9.58 \mathrm{e}-02$ & $9.42 \mathrm{e}-01$ & $3.21 \mathrm{e}+00$ & $7.97 \mathrm{e}-01$ & $0 / 30$ \\
\hline SGA-20S & $2.02 \mathrm{e}-01$ & $1.39 \mathrm{e}+00$ & $3.34 \mathrm{e}+00$ & $1.04 \mathrm{e}+00$ & $0 / 30$ \\
\hline SGA-20R & $1.43 \mathrm{e}-01$ & $1.14 \mathrm{e}+00$ & $3.03 \mathrm{e}+00$ & $8.93 \mathrm{e}-01$ & $0 / 30$ \\
\hline SaNSDE & $7.16 \mathrm{e}-08$ & $2.82 \mathrm{e}-03$ & $4.98 \mathrm{e}-02$ & $1.01 \mathrm{e}-02$ & $27 / 30$ \\
\hline SaNSDE+ & $\mathbf{5 . 4 0 e - 0 8}$ & $\mathbf{8 . 9 6 e - 0 8} \dagger$ & $\mathbf{1 . 7 1 e - 0 7}$ & $\mathbf{3 . 1 7 e - 0 8}$ & $\mathbf{3 0 / 3 0}$ \\
\hline
\end{tabular}

" $\uparrow$ " indicates that the result is significantly better than all others by the Wilcoxon rank sum test with $p<0.001$

TABLE VI

BEST PARAMETER SOLUTION FOUND BY ALL FEP VARIANTS (FEP-*), SGA VARIANTS (SGA-*), SANSDE, AND SANSDE+

\begin{tabular}{|l|l|l|l|l|}
\hline ID/Given & FEP $-\star$ & SGA $-\star$ & SaNSDE & SaNSDE + \\
\hline$x_{1} / 0.13$ & 0.129639 & 0.130549 & 0.13 & 0.13 \\
\hline$x_{2} / 0.04$ & 0.0391089 & 0.0687786 & 0.04 & 0.04 \\
\hline$x_{3} / 0.90$ & 0.922775 & 0.530787 & 0.90 & 0.90 \\
\hline$x_{4} / 0.90$ & 0.927748 & 0.563686 & 0.90 & 0.90 \\
\hline$x_{5} / 0.13$ & 0.130192 & 0.129786 & 0.13 & 0.13 \\
\hline$x_{6} / 0.13$ & 0.132754 & 0.133208 & 0.13 & 0.13 \\
\hline$x_{7} / 0.13$ & 0.13014 & 0.12998 & 0.13 & 0.13 \\
\hline$x_{8} / 0.04$ & 0.0372729 & 0.10196 & 0.0399999 & 0.0399999 \\
\hline$x_{9} / 0.80$ & 0.856409 & 0.249166 & 0.800001 & 0.800001 \\
\hline$x_{10} / 0.90$ & 0.96463 & 0.294441 & 0.900001 & 0.900001 \\
\hline
\end{tabular}

Optimal parameter values are given in the "Given" column.

early and made little progress thereafter, but SaNSDE+ was able to keep achieving improvements throughout the evolution process. The results of this part support the conclusion that is drawn based on benchmark functions [27] that SaNSDE+ is one of the state-of-the-art EA for numerical optimization.

Besides the final results, simplicity and the ease of use are also important issues when evaluating the practicability of an algorithm, because it is critical for the user to reobtain the reported performance. For EAs, the practicability is basically reflected by the number of control parameters and the sensitivity of EAs' performance with respect to parameter settings. It often takes 


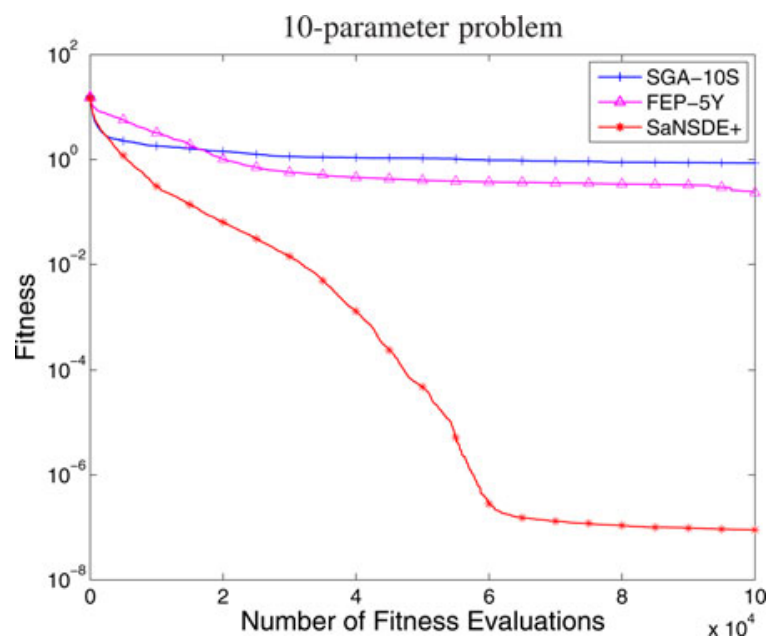

Fig. 7. Evolution curves of SGA-10S, FEP-5Y, and SaNSDE+ for the tenparameter problem. All results have been averaged over 30 runs.

a lot of time to tune these parameters for a specific problem. Among the three compared algorithms, SaNSDE + has the least number of parameters, i.e., two parameters NP and $p$. Since population size NP is a common parameter for all population based algorithms, SaNSDE + actually has only one parameter to be predefined. Moreover, the performance of SaNSDE is not very sensitive to the parameter $p$, which is used to select "lpbest." The evidence can be found by analyzing the results of SaNSDE in Table V, and Fig. 6. The only difference between SaNSDE and SaNSDE + is that the parameter $p$ is introduced in the latter one for selecting "Ipbest." However, the previous SaNSDE, in which the parameter $p$ is degenerated by using the best individual as "lpbest," still achieved much better results than all the SGA and FEP variants. The use of $p$ is only helpful to improve the convergence speed and the ratio of successful runs of SaNSDE. For SGA, which is one of the earliest proposed EAs, has the maximum number of control parameters. All these parameters have to be tuned manually since no adaptation or self-adaptation mechanism is used to adapt them. FEP is a more advanced EA than SGA. It adopts a self-adaptive strategy to control its search step size during evolution [30]. However, the initial step size, which is problem dependent, still needs to be determined in advance. Although the performance of SGA and FEP could be improved by parameter tuning, it is still difficult for them to identify the focused building parameters correctly. In contrast, we proposed several adaptation mechanisms in SaNSDE + to adapt almost all of its control parameters automatically, which would make it much easier for user to solve their own applications expediently.

\section{Validation Using Large Dataset and Comparison With State-of-the-Art EAs}

All the results that are presented in Sections V-B and V-C were achieved based on the HAMBase simulation with 1-mo input data, which might not be sufficient to build highly reliable conclusions. The reason why we first used only 1-mo data to evaluate EAs performance is that the simulation of the HAMBase model is computationally expensive, especially for large
TABLE VII

Comparison on the Ten-Parameter Problem Using the 9-Mo Dataset

\begin{tabular}{|l|c|c|c|c|c|}
\hline & FEP-5Y & SGA-10S & CLPSO & JADE & SaNSDE + \\
\hline Best & $1.15 \mathrm{e}-01$ & $4.92 \mathrm{e}-01$ & $1.38 \mathrm{e}-01$ & $\mathbf{6 . 4 5 e - 0 8}$ & $1.03 \mathrm{e}-07$ \\
\hline Median & $2.51 \mathrm{e}-01$ & $9.41 \mathrm{e}-01$ & $2.12 \mathrm{e}-01$ & $1.97 \mathrm{e}-01$ & $\mathbf{5 . 7 3 e - 0 7}$ \\
\hline Worst & $2.27 \mathrm{e}+00$ & $3.09 \mathrm{e}+00$ & $4.46 \mathrm{e}-01$ & $3.87 \mathrm{e}+00$ & $\mathbf{9 . 1 7 e - 0 4}$ \\
\hline Mean & $3.22 \mathrm{e}-01$ & $1.01 \mathrm{e}+00$ & $2.57 \mathrm{e}-01$ & $3.10 \mathrm{e}-01$ & $\mathbf{7 . 4 5 e - 0 5} \dagger$ \\
\hline Std & $3.86 \mathrm{e}-01$ & $6.73 \mathrm{e}-01$ & $1.04 \mathrm{e}-01$ & $6.79 \mathrm{e}-01$ & $\mathbf{2 . 3 2 e - 0 4}$ \\
\hline SR & $0 / 30$ & $0 / 30$ & $0 / 30$ & $3 / 30$ & $23 / 30$ \\
\hline
\end{tabular}

" $\nmid$ " indicates that the result is significantly better than all others by the

Wilcoxon rank sum test with $p<0.001$.

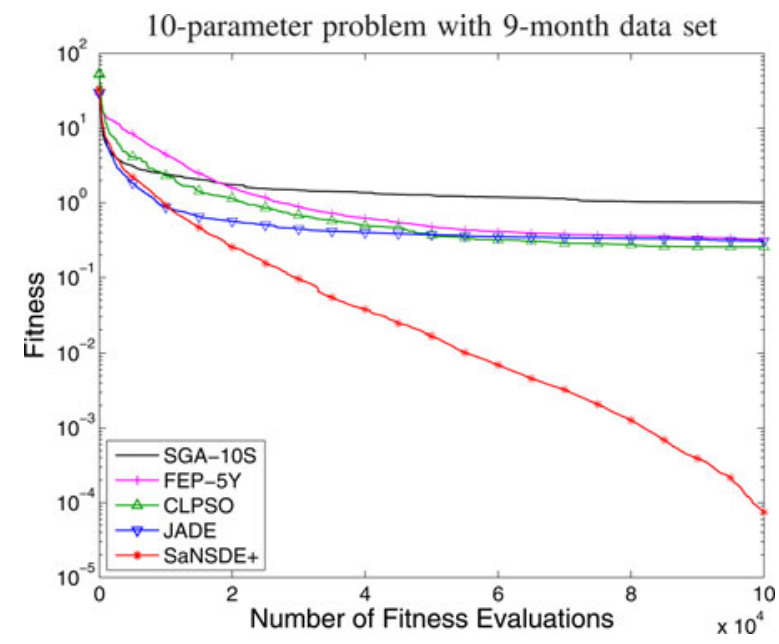

Fig. 8. Evolution curves of SGA-10S, FEP-5Y, and SaNSDE+ for the tenparameter problem using the 9-mo dataset. All results have been averaged over 30 runs.

input datasets. For example, it takes about 1 week to simulate HAMBase with the 9-mo dataset on one Intel 2.4-GHz CPU core for each run of each algorithm. Therefore, to save time, it would be good to first evaluate all the candidate EAs with small dataset, and then verify only the best ones with a sufficiently large dataset. Dataset with a size of 9 mo is often regarded as acceptable because it ensures that the building sees the two extremes of the seasonal changes [53].

Moreover, to support the claim that SaNSDE+ is efficient for such kind of problems, it may not sufficient to compare its efficacy with only classical EAs, i.e., SGA and FEP. Because in recent years EC has greatly advanced, and many new EA variants have been proposed to improve the efficacy of classical EAs. Therefore, we should further compare SaNSDE+ with some state-of-the-art EAs.

In this part, we further validated the best representatives (i.e., FEP-5Y, SGA-10S, and SaNSDE+) of the tested three kinds of EAs by using the simulation of HAMBase with 9-mo dataset. We also compared the results with two recently proposed and highly competitive EAs in the area of global optimization. The first one is CLPSO [31], which is one of the best variants of PSO [54]. The other one is the most recently proposed DE variant, i.e., JADE [32]. Both CLPSO and JADE have been evaluated extensively using benchmark functions.

The simulation data were taken from a time period of 9 mo starting from Oct. 1, 1998. The data were also collected at discrete hourly time steps. The parameter settings of FEP5Y, SGA-10S, and SaNSDE+ are the same as that used in 
Section V-C. The parameters of CLPSO and JADE were set to the suggested values in their literature works [31] and [32]. The results of 30 independent runs are summarized in Table VII. We carried out the results in parallel by using the "Blue BEAR" cluster of the University of Birmingham.

For the results in Table VII, SaNSDE+ still outperformed FEP-5Y and SGA-10S significantly, which is consistent with the previous conclusion drawn by using 1-mo dataset. Although the newly tested CLPSO is quite stable and is better than both FEP$5 \mathrm{Y}$ and SGA-10S, it is still not comparable with SaNSDE+. JADE got the best fitness value among all the tested EAs. However, its success rate is very low ( $\mathrm{SR}=3 / 30)$, and hence, its overall performance is affected greatly.

Comparing with the results in Table V, it can be found that the performance of SaNDE+, FEP-5Y, and SGA-10S deteriorated against the increase in dataset size. This is unsurprising because we used the same number of FEs to handle a much larger dataset, for which we need to fit the building model with a longer output sequence. The situation might be different if we optimize the more difficult problem with more number of FEs. The claim can be confirmed by looking at the evolution curves in Fig. 8 . It seems SaNSDE + is not converged and still has a lot of potential to obtain better results. This also explained why the ratio of successful runs dropped from $\mathrm{SR}=30 / 30$ to $\mathrm{SR}=23 / 30$. SaNSDE + may need more time budget to converge for the 9-mo problem. Even without increasing the number of FEs, the ratio of successful runs of SaNSDE+ is still rather high. Therefore, basically, it can be concluded that the proposed SaNSDE+ is capable of solving the parameter identification problem with large datasets as well.

\section{DISCUSSIONS AND CONCLUSION}

In this paper, a study of state-of-the-art EAs, i.e., SaNSDE and SaNSDE+, as applied to a building thermal model parameter identification problem, which is typically very difficult to solve using traditional optimization methods, has been presented. Optimization problems with six and ten objective parameters were formulated based upon the HAMBase building simulation model. A fitness function has been designed to measure the quality of identified parameter values by comparing the difference between the output of building model and that of the target building. SaNSDE + is a new and improved variant of SaNSDE, which employs a more efficient mutation strategy to achieve a superior convergence speed.

The results that have been described in this paper suggest that the adopted EAs, especially SaNSDE + , are very effective for parameter identification problems. All the selected parameters of the building model were identified accurately. The results of SaNSDE + are significantly better than that of two other wellknown EAs, i.e., SGA and FEP.

Unfortunately, resource constraints limited this study to building simulations. In the future, we will investigate this method applied to real buildings. Once we show this method can be successfully applied, it could be integrated with existing smart energy metering services to monitor the usage of building energy for temperature regulation.
The optimization approach that has been developed in this paper is taking the building model as a black box. It should not be model dependent and is expected to work for other models as well. Therefore, extending the proposed method to such parameter identification problems in other complicated models (e.g., neural models [19]) could also be interesting future research directions.

\section{REFERENCES}

[1] L. Pérez-Lombard, J. Ortiz, and C. Pout, "A review on buildings energy consumption information," Energy Build., vol. 40, no. 3, 2008.

[2] M. D. Felice and X. Yao, "Short-term load forecasting with neural network ensembles: A comparative study," IEEE Comput. Intell. Mag., vol. 6, no. 3, pp. 47-56, Aug. 2011.

[3] M. R. Amin-Naseri and A. R. Soroush, "Combined use of unsupervised and supervised learning for daily peak load forecasting," Energy Convers. Manage., vol. 49, no. 6, pp. 1302-1308, 2008.

[4] S. Tzafestas and E. Tzafestas, "Computational intelligence techniques for short-term electric load forecasting," J. Intell. Robot. Syst., vol. 31, no. 1 , pp. 7-68, 2001.

[5] K. Metaxiotis, A. Kagiannas, D. Askounis, and J. Psarras, "Artificial intelligence in short term electric load forecasting: A state-of-the-art survey for the researcher," Energy Convers. Manage., vol. 44, no. 9, pp. 1525-1534, 2003.

[6] H. Steinherz, C. Pedreira, and R. Castro, "Neural networks for short-term load forecasting: A review and evaluation," IEEE Trans. Power Syst., vol. 16, no. 1, pp. 44-55, Feb. 2001.

[7] N. Amjady, "Short-term hourly load forecasting using time-series modeling with peak load estimation capability," IEEE Trans. Power Syst., vol. 16, no. 4, pp. 798-805, Nov. 2001.

[8] J. F. Karlsson and B. Moshfegh, "Energy demand and indoor climate in a low energy building changed control strategies and boundary conditions," Energy Build., vol. 38, no. 4, pp. 315-326, 2006.

[9] H. Breesch, A. Bossaer, and A. Janssens, "Passive cooling in a low-energy office building," Solar Energy, vol. 79, no. 6, pp. 682-696, 2005.

[10] DOE-2 Engineering Manual, version 2.1C, Lawrence Berkeley Laboratory, Berkeley, CA, 1982.

[11] Energy Plus. (May 2011). [Online]. Available: http://www.eere.energy. gov/buildings/energyplus/

[12] M. Macias, A. Mateo, M. Schuler, and E. M. Mitre, "Application of night cooling concept to social housing design in dry hot climate," Energy Build., vol. 38, no. 9, pp. 1104-1110, 2006.

[13] T. Ayata and O. Yildiz, "Investigating the potential use of natural ventilation in new building designs in Turkey," Energy Build., vol. 38, no. 8, pp. 959-963, 2006.

[14] M. L. Persson, A. Roos, and M. Wall, "Influence of window size on the energy balance of low energy houses," Energy Build., vol. 38, no. 3, pp. 181-188, 2006.

[15] M. de Wit, HAMBase: Heat, Air and Moisture Model for Building and Systems Evaluation. Eindhoven, the Netherlands: the Eindhoven University Press, 2006. ISBN: 90-6814-601-7.

[16] A. W. M. van Schijndel, Integrated Heat Air and Moisture Modeling and Simulation. Eindhoven, the Netherlands: Adrianus Wilhelmus Maria van Schijndel, 2007. ISBN: 9789068146042.

[17] J. Nocedal and S. J. Wright, Numerical Optimization. New York: Springer-Verlag, 1999

[18] R. Sarker, M. Mohammadian, and X. Yao, Evolutionary Optimization. Norwell, MA: Kluwer, 2002.

[19] W. Van Geit, E. De Schutter, and P. Achard, "Automated neuron model optimization techniques: A review," Biological Cybern., vol. 99, no. 4, pp. 241-251, 2008.

[20] M. Tang and X. Yao, "A memetic algorithm for VLSI floorplanning," IEEE Trans. Syst., Man Cybern. B, Cybern., vol. 37, no. 1, pp. 62-69, Feb. 2007.

[21] J. A. Wright, "HVAC optimisation studies: Sizing by genetic algorithm," Build. Serv. Eng. Res. Technol., vol. 17, no. 1, pp. 7-14, 1996.

[22] J. A. Wright, H. A. Loosemore, and R. Farmani, "Optimization of building thermal design and control by multicriterion genetic algorithm," Energy Build., vol. 34, no. 9, pp. 959-972, 2002. 
[23] J. A. Wright, Y. Zhang, P. Angelov, V. Hanby, and R. Buswell, "Evolutionary synthesis of HVAC system configurations: Algorithm development," HVAC\&R Res., vol. 14, no. 1, pp. 33-55, 2008.

[24] S. Wang and X. Xu, "Simplified building model for transient thermal performance estimation using GA-based parameter identification," Int. J. Therm. Sci., vol. 45, no. 4, pp. 419-432, 2006.

[25] S. Wang and X. Xu, "Parameter estimation of internal thermal mass of building dynamic models using genetic algorithm," Energy Convers. Manage., vol. 47, no. 13-14, pp. 1927-1941, 2006.

[26] X. Xu and S. Wang, "Optimal simplified thermal models of building envelope based on frequency domain regression using genetic algorithm," Energy Build., vol. 39, no. 5, pp. 525-536, 2007.

[27] Z. Yang, K. Tang, and X. Yao, "Self-adaptive differential evolution with neighborhood search," in Proc. IEEE Congr. Evolut. Comput., 2008, pp. 1110-1116.

[28] Z. Yang, K. Tang, and X. Yao, "Large scale evolutionary optimization using cooperative coevolution," Inf. Sci., vol. 178, no. 15, pp. 2985-2999, 2008.

[29] M. Mitchell, An Introduction to Genetic Algorithms. Cambridge, MA: MIT Press, 1998. ISBN: 978-0-262-63185-3.

[30] X. Yao, Y. Liu, and G. Lin, "Evolutionary programming made faster," IEEE Trans. Evol. Comput., vol. 3, no. 2, pp. 82-102, Jul. 1999.

[31] J. J. Liang, A. K. Qin, P. N. Suganthan, and S. Baskar, "Comprehensive learning particle swarm optimizer for global optimization of multimodal functions," IEEE Trans. Evol. Comput., vol. 10, no. 3, pp. 281-295, Jun. 2006.

[32] J. Zhang and A. C. Sanderson, "JADE: Adaptive differential evolution with optional external archive," IEEE Trans. Evol. Comput., vol. 13, no. 5, pp. 945-958, Oct. 2009.

[33] HAMLab codes. (May 2011). [Online]. Available: http://sts.bwk.tue. $\mathrm{nl} / \mathrm{hamlab} /$

[34] K. E. Vugrin, "On the effect of numerical noise in simulation-based optimization" Ph.D. dissertation, Dept. Math., Virginia Polytechnic Institute and State University, Blacksburg, VA, 2005.

[35] Y. Jin and J. Branke, "Evolutionary optimization in uncertain environments: A survey," IEEE Trans. Evol. Comput., vol. 9, no. 3, pp. 303-317, Jun. 2005.

[36] R. Storn and K. Price, "Differential evolution-A simple and efficient heuristic for global optimization over continuous spaces," J. Global Optim., vol. 11, no. 4, pp. 341-359, 1997.

[37] K. Price, R. Storn, and J. Lampinen, Differential Evolution: A Practical Approach to Global Optimization. New York: Springer-Verlag, 2005.

[38] J. Vesterstrom and R. Thomsen, "A comparative study of differential evolution, particle swarm optimization, and evolutionary algorithms on numerical benchmark problems," in Proc. IEEE Congr. Evol. Comput., 2004, vol. 2, pp. 1980-1987.

[39] Z. Yang, K. Tang, and X. Yao, "Scalability of generalized adaptive differential evolution for large-scale continuous optimization," Soft Comput., vol. 15, no. 11, pp. 2141-2155, 2011.

[40] R. Storn, "System design by constraint adaptation and differential evolution," IEEE Trans. Evol. Comput., vol. 3, no. 1, pp. 22-34, Apr. 1999.

[41] C.-H. Chen, C.-J. Lin, and C.-T. Lin, "Nonlinear system control using adaptive neural fuzzy networks based on a modified differential evolution," IEEE Trans. Syst., Man Cybern. C, Appl.Rev., vol. 39, no. 4, pp. 459-473, Jul. 2009

[42] A. K. Qin, V. L. Huang, and P. N. Suganthan, "Differential evolution algorithm with strategy adaptation for global numerical optimization," IEEE Trans. Evol. Comput., vol. 13, no. 2, pp. 398-417, Apr. 2009.

[43] Z. Yang, J. Zhang, K. Tang, X. Yao, and A. C. Sanderson, "An adaptive coevolutionary differential evolution algorithm for large-scale optimization," in Proc. IEEE Congr. Evol. Comput., 2009, pp. 102-109.

[44] D. Zaharie, "Critical values for the control parameters of differential evolution algorithms," in Proc. 8th Int. Conf. Soft Comput., 2002, pp. 62-67.

[45] R. Gamperle, S. D. Muller, and P. Koumoutsakos, "A parameter study for differential evolution," in Proc. WSEAS Int. Conf. Adv. Intell. Syst., Fuzzy Syst., Evol. Comput., 2002, pp. 293-298.

[46] Z. Yang, J. He, and X. Yao, "Making a difference to differential evolution," in Advances in Metaheuristics for Hard Optimization, Z. Michalewicz and P. Siarry, Eds. New York: Springer-Verlag, 2008, pp. 397-414.

[47] J. Brest, S. Greiner, B. Boskovic, M. Mernik, and V.Žumer, "Self-adapting control parameters in differential evolution: A comparative study on numerical benchmark problems," IEEE Trans. Evol. Comput., vol. 10, no. 6, pp. 646-657, Dec. 2006.

[48] M. Omran and A. P. Engelbrecht, "Differential evolution for integer programming problems," in Proc. IEEE Congr. Evol. Comput., 2007, pp. 2237-2242.
[49] B. Li, J. Lin, and X. Yao, "A novel evolutionary algorithm for determining unified creep damage constitutive equations," Int. J. Mech. Sci., vol. 44, no. 5, pp. 987-1002, 2002.

[50] J. Li, X. Yao, C. Frayn, H. Khosroshahi, and S. Raychaudhury, "An evolutionary approach to modeling radial brightness distributions in elliptical galaxies," in Parallel Problem Solving from Nature—PPSN VIII. New York: Springer-Verlag, 2004, pp. 591-601.

[51] A. Chipperfield and P. Fleming. (1995). The MATLAB genetic algorithm toolbox. in Proc. IEE Colloquium Appl. Control Tech. Using MATLAB. [Online]. pp. 10/1-10/4. Available: http://www.shef.ac.uk/acse/research/ecrg/gat.html

[52] K.-H. Liang, X. Yao, and C. Newton, "Dynamic Control of Adaptive Parameters in Evolutionary Programming," in Proc. 2nd Asia-Pacific Conf. Simulated Evol. Learn., Selected Papers, 1999, vol. 1585, pp. 41-49.

[53] J. F. Kreider, Handbook of Heating, Ventilation, and Air Conditioning. Boca Raton, FL: CRC Press, 2001.

[54] J. Kennedy and R. C. Eberhart, "Particle swarm optimization," in Proceedings of IEEE International Conference on Neural Networks. vol. 4, Piscataway, NJ: IEEE Press, 1995, pp. 1942-1948.

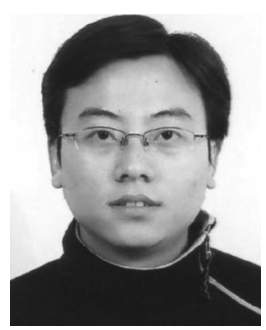

Zhenyu Yang (S'07-M'10) received the B.Eng. and $\mathrm{Ph} . \mathrm{D}$. degrees from the University of Science and Technology of China, Hefei, Anhui, China, in 2005 and 2010, both in computer science. From October 2008 to September 2009, he was the visiting Ph.D. student with the School of Computer Science, University of Birmingham, Birmingham, U.K., supported by the China Scholarship Council (CSC).

From July 2010 to October 2011, he was a Lecturer with the Department of Computer Science and Technology, East China Normal University, Shanghai, China. He is currently a Lecturer with the Department of Management Science and Engineering, College of Information Systems and Management, National University of Defense Technology, Changsha, China. His research interests include metaheuristics, such as evolutionary algorithms for global optimization, large-scale optimization, and various real-world applications.

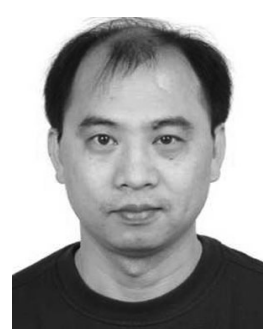

Xiaoli Li received the B.S.E. and M.S.E. degrees from Kun-Ming University of Science and Technology, and the Ph.D degree from Harbin Institute of Technology, China, in 1992, 1995, and 1997, respectively, all in mechanical engineering.

From April 1998 to Oct. 2003, he was a Research Fellow with the Department of Manufacturing Engineering, City University of Hong Kong, of the Alexander von Humboldt Foundation with the Institute for Production Engineering and Machine Tools, Hannover University, Germany, a Postdoctoral fellow with the Department of Automation and Computer-Aided Engineering, Chinese University of Hong Kong. From 2003 to 2009, he was a Research fellow with the Center of Excellence for Research in Computational Intelligence and Applications (CERCIA), School of Computer Science, University of Birmingham, Birmingham, U.K. He is currently the Professor and Head of Department of Automation, Institute of Electrical Engineering, Yanshan University, China. Since 2011, he has been a Full Professor with the National Key Laboratory of Cognitive Neuroscience and Learning, Beijing Normal University, China. His research interests include neural engineering, computation intelligence, signal processing and data analysis, monitoring system, and manufacturing system. His current research projects include National Science Fund for Distinguished Young Scholars, National Natural Science Foundation, Program for New Century Excellent Talents in University, and Hebei Science Fund for Distinguished Young Scholars. 


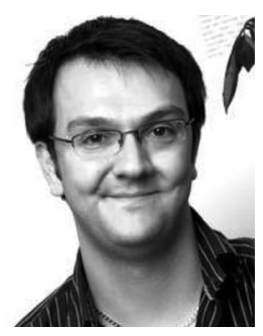

Chris P. Bowers received the B.Sc. degree in computer science and physics from Keele University in 2001, and the M.Sc. degree in natural computation and the Ph.D. degree in computer science in 2002 and 2006, respectively, both from the University of Birmingham.

He is currently a Research Fellow with the Centre Excellence for Research in Computational Intelligence and Applications (CERCIA), School of Computer Science at the University of Birmingham, Birmingham, U.K. His main research interests include applications of computational intelligence and robust optimization particularly in the context of complex and dynamic environments and intelligent adaptive interfaces.

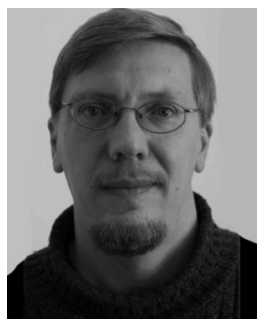

Thorsten Schnier (M'00) received the Dipl.Ing degree in electrical engineering from the University of Hannover, Germany, and the Ph.D. degree from the University of Sydney, Sydney, Australia.

He joined the School of Computer Science at the University of Birmingham in 1999. He is now a Research Fellow in the Centre of Excellence for Research in Computational Intelligence and Applications (CERCIA) and part of the Natural Computation Group in the School of Computer Science, As part of CERCIA, he has worked with a large number of industrial partners, and has developed extensive experience with cross-disciplinary work, in particular with the application of computational intelligence to industrial problems. His research grants, published work and invited talks include a broad range of applications of computational intelligence, including optimization of electronic circuits, turbine blades and automotive engine control, as well as machine creativity, modelling of electricity markets, and building modelling. The work has also lead to two patents in design optimization.

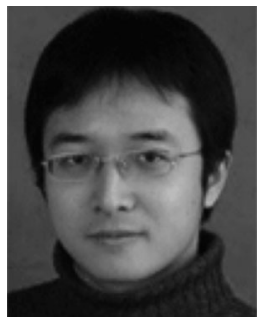

Ke Tang (S'05-M'07) received the B.Eng. degree from the Huazhong University of Science and Technology, Wuhan, China, in 2002 and the Ph.D. degree from the School of Electrical and Electronic Engineering, Nanyang Technological University, Singapore, in 2007.

In 2007, he joined the Nature Inspired Computation and Applications Laboratory (NICAL), School of Computer Science and Technology, University of Science and Technology of China, Hefei, China, where he was promoted to Professor in 2011. He is the author/coauthor of more than 50 refereed publications. His main research interests include machine learning, evalutionary computation, data mining, metaheuristic algorithms, and real-world applications.

Dr. Tang is an Associate Editor of IEEE COMPUTATIONAL INTELLIGENCE MAGAZINE and the Chair of the IEEE Task Force on Large Scale Global Optimization. Task Froce on Collaborative Learning and Optimization. He served as a Program Co-Chair of 2010 IEEE Congress on Evolutionary Computation, held in Barcelona, Spain.

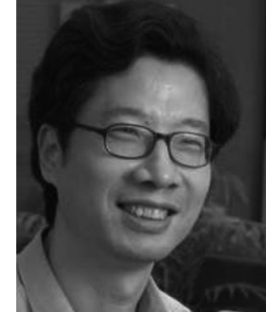

Xin Yao (M'91-SM'96-F'03) received the B.S. degree from the University of Science and Technology of China (USTC), Hefei, Anhui, China, in 1982, the M.S. degree from the North China Institute of Computing Technology, Beijing, China, in 1985, and the $\mathrm{Ph} . \mathrm{D}$. degree from USTC, in 1990, all in computer science.

From 1985 to 1990, he was an Associate Lecturer and Lecturer with the USTC, while working toward the Ph.D. degree focussed on simulated annealing and evolutionary algorithms. In 1990, he was a Postdoctoral Fellow with the Computer Sciences Laboratory, Australian National University, Canberra, Australia, where he continued his work on simulated annealing and evolutionary algorithms. In 1991, he was with the Knowledge-Based Systems Group, Commonwealth Scientific and Industrial Research Organization Division of Building, Construction and Engineering, Melbourne, Australia, where he worked primarily on an industrial project on automatic inspection of sewage pipes. In 1992, he returned to Canberra to take up a Lectureship with the School of Computer Science, University College, University of New South Wales, Australian Defense Force Academy, Sydney, Australia, where he was later promoted to a Senior Lecturer and Associate Professor. He moved to the University of Birmingham, Edgbaston, Birmingham, U.K., where he became a Professor (Chair) of Computer Science on April 1, 1999. He is currently the Director of the Center of Excellence for Research in Computational Intelligence and Applications, School of Computer Science (CERCIA), University of Birmingham. He is currently also a Changiiang (Visiting) Chair Professor (Cheung Kong Scholar) with the Nature Inspired Computation and Applications Laboratory (NICAL), School of Computer Science and Technology, USTC. He has given more than 50 invited keynote and plenary speeches at conferences and workshops worldwide. He has more than 300 refereed publications. His major research interests include evolutionary artificial neural networks, automatic modularization of machine learning systems, evolutionary optimization, constraint-handling techniques, computational time complexity of evolutionary algorithms, coevolution, iterated prisoners dilemma, data mining, and real-world applications.

Dr. Yao was the Editor-in-Chief of the IEEE Transactions on Evolutionary Computation from 2003 to 2008, an Associate Editor or Editorial Board Member of 12 other journals, and the Editor of the World Scientific Book Series on Advances in Natural Computation. He received the Presidents Award for Outstanding Thesis by the Chinese Academy of Sciences for his Ph.D. work on simulated annealing and evolutionary algorithms in 1989 . He was the recipient of the 2001 IEEE Donald G. Fink Prize Paper Award for his work on evolutionary artificial neural networks. 